\title{
TUBERCULOSIS QUE IMITA CÁNCER: CASOS DERIVADOS AL INSTITUTO NACIONAL DE ENFERMEDADES NEOPLÁSICAS, LIMA-PERÚ
}

\author{
Juliana R. Villena-Suarez ${ }^{1, a}$, William Vicente ${ }^{1, b}$, Luis Taxa ${ }^{1, c}$, Luis Cuéllar ${ }^{1, d}$, Maria T. Nuñez-Butrón ${ }^{1, e}$, \\ Valeria Villegas ${ }^{1, f}$, Miluska Castillo ${ }^{1,9}$ Carlos A. Castañeda ${ }^{1, h}$
}

\begin{abstract}
RESUMEN
La tuberculosis (TB) es un importante problema de salud pública que debido a la variabilidad clínica de su presentación, puede confundirse con una malignidad. El objetivo del estudio fue identificar las características clínico radiológicas y describir la metodología que permitió llegar al diagnóstico de TB en pacientes derivados con presunción diagnóstica de cáncer al Instituto Nacional de Enfermedades Neoplásicas (INEN) entre 2014 y 2016 . Se incluyeron 170 pacientes (52,4 \% hombres) con edad promedio de 41,1 años, $18 \%$ presentaron antecedentes de contacto con TB y un 5,9\% tuvo previamente la enfermedad. La TB fue pulmonar en $22,4 \%$ y extrapulmonar en $77,7 \%$ de los pacientes. Los síntomas más frecuentes fueron respiratorios, tumoración, pérdida de peso y neurológicos. Los diagnósticos oncológicos descartados con mayor frecuencia fueron linfoma, cáncer pulmonar y cerebral. Las lesiones que sugerían una neoplasia indicaron un estadio clínico avanzado en el $63,5 \%$. Se concluye que los síntomas e imágenes asociados a TB pueden confundirse con neoplasias malignas.
\end{abstract}

Palabras clave: Mycobacterium tuberculosis; Neoplasias; Signos y síntomas; Diagnóstico (fuente: DeCS BIREME).

\section{TUBERCULOSIS THAT MIMICS CANCER: CASES REFERRED TO THE NATIONAL INSTITUTE OF NEOPLASTIC DISEASES, LIMA-PERU}

\begin{abstract}
Tuberculosis (TB) is a major public health problem that, due to the clinical variability of its presentation, can be confused with cancer. The aim of this study was to identify the clinical-radiological characteristics and to describe the methodology that allowed to achieve a TB diagnosis in patients referred to the National Institute of Neoplastic Diseases (INEN) with a presumed diagnosis of cancer between 2014 and 2016. The study included 170 patients $(52.4 \%$ men) with an average age of 41.1 years; $18 \%$ presented a history of contact with TB, and $5.9 \%$ had had the disease previously. The TB was pulmonary in $22.4 \%$ and extrapulmonary in $77.7 \%$ of patients. The most frequent symptoms were respiratory, tumor, weight loss, and neurological. The cancer diagnoses most frequently discarded were lymphoma, lung cancer, and brain cancer. The lesions that suggested a neoplasm indicated an advanced clinical stage in $63.5 \%$. Therefore, it follows that the symptoms and images associated with TB can be confused with malignant neoplasms.
\end{abstract}

Keywords: Mycobacterium tuberculosis; Neoplasms; Signs and symptoms; Diagnosis (source: MeSH NLM).

\section{INTRODUCCIÓN}

La tuberculosis (TB) es un problema de salud pública en el Perú donde se han notificado 30988 casos y 87,6 casos nuevos por cada 100 mil habitantes en 2015. Sin embargo, existe un porcentaje importante de casos que no se notifican y la Organización Mundial de la Salud los estima en 7000 para el $2015^{(1,2)}$. La TB pulmonar (TBP) representa el $70 \%$ de los casos y se asocia a síntomas de tos, hemoptisis y pérdida de peso, así como, a imágenes de cavidades pulmonares de paredes gruesas, similares a las halladas en el cáncer de pulmón ${ }^{(3-9)}$. El espectro clínico de la TB extrapulmonar sigue siendo incierto y muchas veces el único indicio será la identificación de granulomas necrotizantes en una biopsia ${ }^{(10-17)}$.

El diagnóstico de cáncer en estadios avanzados es frecuente en el Perú, convirtiéndose en un problema de salud pública, y dada la alta prevalencia de TB pueden ser frecuentemente confundidas. Sin embargo, no existe información publicada en este campo en nuestro país ${ }^{(1)}$.

\footnotetext{
Instituto Nacional de Enfermedades Neoplásicas

a Médico cirujano; ${ }^{\mathrm{b}}$ médico especialista en anatomía patológica; ${ }^{\mathrm{c}}$ médico especialista en patología oncológica, doctor en Medicina; ${ }^{\mathrm{d}}$ médico especialista en enfermedades infecciosas y tropicales; ${ }^{\mathrm{e}}$ médico especialista en anatomía patológica, magíster en medicina; ${ }^{\mathrm{f}}$ licenciada en Biología; ${ }^{\mathrm{g}}$ toxicóloga; ${ }^{\mathrm{h}}$ especialista en oncología clínica, magíster en genética y biología celular, magíster en oncología avanzada.

Recibido: 14/11/2017 Aprobado: 07/03/2018 En línea: 05/04/2018
}

Citar como: Villena-Suarez JR, Vicente W, Taxa L, Cuéllar L, Nuñez-Butrón MT, Villegas V, Castillo M, Castaneda CA. Tuberculosis que imita cáncer: casos derivados al Instituto Nacional de Enfermedades Neoplásicas, Lima-Perú. Rev Peru Med Exp Salud Publica. 2018;35(1):77-83. doi: 10.17843/rpmesp.2018.351.3602. 
El objetivo del presente estudio fue identificar las características clínico radiológicas que sugirieron la malignidad y describir la metodología que permitió llegar al diagnóstico del agente infeccioso en los casos de TBP y extrapulmonar que fueron referidos con presunción diagnóstica de cáncer al Instituto Nacional de Enfermedades Neoplásicas (INEN).

\section{EL ESTUDIO}

Se revisó el registro de pacientes atendidos entre enero 2014 y diciembre 2016 en el Laboratorio de Microbiología del INEN que fueron diagnosticados de TB activa. Se consideró casos TB-positivos cuando el análisis histológico describió granulomas, el examen microbiológico evidenciaba bacilos (baciloscopia) en tinción Ziehl-Neelsen, el cultivo en medio líquido con Middlebrook y en medio sólido con Löwenstein-Jensen fue positivo a $M$. tuberculosis y/o la reacción en cadena de polimerasa (PCR) fue positiva para el gen IS6110. Se consideró como criterio de exclusión el diagnóstico concurrente de neoplasia maligna.

Se revisaron retrospectivamente las historias clínicas, los informes de las imágenes (radiografías, tomografía computarizada (TC), ecografías y resonancia magnética (RM)), los informes de anatomía patológica y los informes del laboratorio de microbiología. Adicionalmente, se revisó prospectivamente las imágenes de las lesiones extrapulmonares en casos con imágenes disponibles en el archivo del departamento de radiología del instituto.

La información obtenida de las historias clínicas se registró en forma codificada en el programa Microsoft Excel 2013. El análisis se realizó utilizando el programa SPSS 17,0 (SPSS, Inc., Chicago, IL, EE. UU.).

Este proyecto obtuvo la aprobación del Comité Institucional Revisor de Protocolos del INEN y toda la metodología de estudio se realizó de acuerdo a la guía STROBE (18).

\section{RESULTADOS}

Se identificaron 170 pacientes con diagnóstico de TB y ausencia de neoplasia que acudieron al INEN. La edad promedio de los pacientes fue de 41,1 años, 89 (52,4 \%) de ellos fueron hombres y 107 (62,9\%) residían en Lima (Lima Metropolitana, provincias y el Callao). Solo dos casos tuvieron antecedentes de infección por VIH. El tiempo promedio de enfermedad fue de 8,1 meses (Tabla 1).

Con relación a los antecedentes de TB de los pacientes, 31 $(18,2 \%)$ pacientes tuvieron contacto con alguna persona con TB y diez (5,9\%) habían sido diagnosticados previamente de TB. Los hallazgos clínicos más frecuentes fueron síntomas respiratorios $(27,7 \%)$, tumoración $(27,7 \%)$ y pérdida de peso $(23,5 \%)$. Los tres hallazgos radiológicos más frecuentes

\section{MENSAJES CLAVE}

Motivación para realizar el estudio. La tuberculosis es un problema frecuente de salud pública en nuestro país y algunos casos pueden ser referidos a servicios oncológicos por su similitud clínica con neoplasias.

Principales hallazgos. Se encontró que la mayoría de casos fueron tuberculosis extrapulmonar y las localizaciones más frecuentes fueron las ganglionares y cerebrales. La mayoría de los casos con tuberculosis impresiona como una neoplasia avanzada.

Implicancias. Es importante tener siempre en cuenta el diagnóstico diferencial entre cáncer y tuberculosis en zonas con alta prevalencia como nuestro país.

al ingreso de los pacientes fueron imagen sugestiva de probable infección en $71(41,8 \%)$, imagen sugestiva de neoplasia maligna en $48(28,2 \%)$ y presencia de adenopatías en 46 casos (27,1\%) (Tabla 1). El diagnóstico diferencial inicial de malignidad realizado por el oncólogo clínico fue de linfoma $(26,5 \%)$, seguido de neoplasia maligna de pulmón $(24,7 \%)$ y de cerebro $(17,7 \%)$, mientras que el $63,5 \%$ de los casos serían sugestivas de una neoplasia avanzada (con metástasis) (Tabla 2).

Se identificaron dos patrones clínicos, TBP (38 pacientes, $22,4 \%$ ) y TB extrapulmonar (132 pacientes, $77,7 \%$ ). Los casos extrapulmonares incluyeron 36 casos de TB ganglionar (21,2 \%), 30 de tuberculoma cerebral $(17,7 \%)$ y 30 de sistémica $(17,7 \%)$ (Tabla 3$)$.

La TB ganglionar se presentó más frecuentemente en la región cervical y axilar; y fue bilateral en $27,8 \%$. La región más frecuentemente comprometida en tuberculoma cerebral fue el lóbulo frontal (10/30), parietal (5/30) y temporal (5/30). Se reportó una lesión única en un 80 \% (24/30) de los casos y se presentaron como lesiones múltiples en 6,7 \%. La mediana del diámetro de la lesión mayor fue $2,7 \mathrm{~cm}$ ( $\mathrm{n}=$ 19), existió edema en $83,3 \%$ (25/30) e infartos vasculares en parénquima en $6,7 \%(2 / 30)$.

Las lesiones óseas se presentaron como lesión única en $62,5 \%$ de los casos; y en metáfisis $(57,1 \%)$ o en diáfisis $(42,9 \%)$. Se localizaron en columna dorsal (2/7), miembro inferior (5/7) y articulación sacrocoxígea (1/7). Las lesiones en el ovario fueron dos, una bilateral y otra en el lado derecho.

Los síntomas y signos más frecuentes en el grupo de TBP fueron síntomas respiratorios $(73,7 \%)$, pérdida de peso $(31,6 \%)$, dolor torácico $(31,6 \%)$, fiebre $(13,2 \%)$, astenia $(13,2 \%)$ y dolor dorsal $(13,2 \%)$.

En el patrón clínico de los pacientes con TB extrapulmonar, predominan la presencia de tumoración $(35,6 \%)$, pérdida de peso $(21,2 \%)$, síntomas neurológicos $(21,2 \%)$, incremento del tamaño de los ganglios (18,2\%), astenia (15,9\%), fiebre $(15,2 \%)$ y dolor abdominal $(11,4 \%)$. 
Tabla 1. Características clínicas de los pacientes del Instituto Nacional de Enfermedades Neoplásicas con diagnóstico de tuberculosis

\begin{tabular}{|c|c|c|}
\hline Característica & $\mathrm{n}=170$ & $\%$ \\
\hline Edad promedio (años) & 41,1 & \\
\hline \multicolumn{3}{|l|}{ Sexo } \\
\hline Femenino & 81 & 47,7 \\
\hline Masculino & 89 & 52,3 \\
\hline Tiempo de enfermedad en meses (promedio) & 8,1 & \\
\hline \multicolumn{3}{|l|}{ Procedencia } \\
\hline Lima & 107 & 62,9 \\
\hline Otro departamento & 63 & 37,1 \\
\hline \multicolumn{3}{|l|}{ Contacto TB } \\
\hline No & 137 & 80,6 \\
\hline $\mathrm{Si}$ & 31 & 18,2 \\
\hline NSR & 2 & 1,2 \\
\hline \multicolumn{3}{|l|}{ Antecedente TB } \\
\hline No & 158 & 92,9 \\
\hline $\mathrm{Si}$ & 10 & 5,9 \\
\hline NSR & 2 & 1,2 \\
\hline \multicolumn{3}{|l|}{ Síntomas* } \\
\hline Respiratorios & 47 & 27,7 \\
\hline Tumoración & 47 & 27,7 \\
\hline Pérdida de peso & 40 & 23,5 \\
\hline Neurológicos & 28 & 16,5 \\
\hline Astenia & 26 & 15,3 \\
\hline Fiebre & 25 & 14,7 \\
\hline Incremento tamaño del ganglio & 24 & 14,1 \\
\hline Otros & 68 & 40,0 \\
\hline \multicolumn{3}{|l|}{ Hallazgos radiológicos* } \\
\hline Probable infección & 71 & 41,8 \\
\hline Sugestivo de neoplasia maligna o metástasis & 48 & 28,2 \\
\hline Adenopatías & 46 & 27,1 \\
\hline Nódulo pulmonar & 30 & 17,7 \\
\hline Nódulos múltiples & 27 & 15,9 \\
\hline Derrame pleural & 25 & 14,7 \\
\hline Edema cerebral & 24 & 14,1 \\
\hline Patrón reticular & 22 & 12,9 \\
\hline Lesión captadora de contraste & 20 & 11,8 \\
\hline Atelectasias & 19 & 11,2 \\
\hline Patrón en árbol en brote & 18 & 10,6 \\
\hline Colecciones abdominales & 14 & 8,2 \\
\hline Vidrio esmerilado & 5 & 2,9 \\
\hline
\end{tabular}

TB: Tuberculosis; NSR: No se reporta.

* Se puede presentar más de una categoría. 
Tabla 2. Neoplasias planteadas en el diagnóstico diferencial de pacientes con tuberculosis del Instituto Nacional de Enfermedades Neoplásicas

\begin{tabular}{lll}
\hline Descripción clínica & $\mathbf{n = 1 7 0}$ & $\mathbf{\%}$ \\
\hline Diagnóstico inicial & & 26,5 \\
\hline Linfoma & 45 & 24,7 \\
\hline NM pulmón & 42 & 17,7 \\
\hline NM cerebro & 30 & 10,0 \\
\hline NM ovario & 17 & 5,3 \\
\hline Sarcoma & 9 & 4,7 \\
\hline Metástasis de primario desconocido & 8 & 2,4 \\
\hline NM laringe & 4 & 1,7 \\
\hline NM testículo & 3 & 1,7 \\
\hline NM gastrointestinal & 3 & 5,3 \\
\hline Otros & 9 & 63,5 \\
\hline Hallazgos radiológicos inicial & & 36,5 \\
\hline Sugestivo de metástasis & 108 & 62 \\
\hline Sugestivo de enfermedad temprana & & \\
\hline NM: Neoplasia maligna & 92 & \\
\hline
\end{tabular}

Según el tipo de muestra solicitada para baciloscopía, las más frecuentes fueron el esputo en 77 casos (34,5\%), la biopsia de la lesión sólida en 49 (22,0 \%), orina en $34(15,2 \%)$, la biopsia de ganglio en $26(11,7 \%)$, lavado bronquial en $20(9,0 \%)$, heces en $11(4,9 \%)$ y líquido pleural en seis $(2,7 \%)$ (Tabla 4$)$.

Se realizaron 192 pruebas de cultivo de los cuales 58 casos (30,2 \%) fueron positivos y 223 pruebas de baciloscopía de los cuales 38 casos $(17,0 \%)$ fueron positivos. El diagnóstico de granuloma a través de histología se realizó en 75 de 89 casos $(84,3 \%)$ y a través de citología en 21 de 57 casos

Tabla 3. Tipo de tuberculosis en pacientes diagnosticados en el Instituto Nacional de Enfermedades Neoplásicas

\begin{tabular}{lcc}
\hline Tipo de tuberculosis & $\mathbf{n = 1 7 0}$ & $\mathbf{\%}$ \\
\hline Pulmonar & 38 & 22,4 \\
\hline Extrapulmonar & 132 & 77,7 \\
\hline Diagnóstico final & & \\
\hline \multicolumn{1}{c}{ TB ganglionar } & 36 & 21,2 \\
\hline Tuberculoma cerebral & 30 & 17,7 \\
\hline TB sistémica & 30 & 17,7 \\
\hline TB ósea y partes blandas & 11 & 6,5 \\
\hline TB enteral y peritoneal & 9 & 5,3 \\
\hline TB genitourinaria & 7 & 4,1 \\
\hline TB pleural & 5 & 2,9 \\
\hline TB laríngea & 3 & 1,8 \\
\hline TB mamaria & 1 & 0,6 \\
\hline
\end{tabular}

TB: tuberculosis.
(36,8 \%). La evaluación mediante PCR se realizó en 34 de estos casos y $27(79,4 \%)$ de ellos fueron positivos para $M$. tuberculosis.

La metodología más común para diagnosticar TB extrapulmonar fue la presencia de granulomas en 60 (45,5\%) casos y la presencia de cultivo positivo en 41 (31,1\%). Se analizó el esputo en 54 casos de TB extrapulmonar, cinco fueron positivos para baciloscopía y 17 positivos para cultivo.

\section{DISCUSIÓN}

Según los resultados del estudio la localización que más frecuentemente se confundió con una neoplasia fue la extrapulmonar $(77,7 \%)$, a pesar que esta supone el $10-20 \%$ del total de TB que padecen los enfermos inmunocompetentes, probablemente debido a que son las lesiones sólidas como los tuberculomas (granulomas tuberculosos) los que tienen mayor probabilidad de ser confundidos con una neoplasia ${ }^{(2,4)}$.

La clínica de la TBP es a menudo inespecífica o asintomática (5\%). Las manifestaciones sistémicas más frecuentes incluyen febrículas, anorexia, sudoración nocturna y baja de peso persistente, además de los síntomas respiratorios como tos, expectoración (mucopurulenta/hemoptoica) o franca hemoptisis ${ }^{(3-9)}$. Se halló que los síntomas más frecuentes fueron respiratorios, pérdida de peso y dolor torácico. Muchos de estos síntomas semejan síntomas de cáncer de pulmón o síndromes paraneoplásicos ${ }^{(7)}$.

Las imágenes de TBP que requieren diagnóstico diferencial con una neoplasia maligna incluyen las lesiones 
Tabla 4. Características de las muestras de baciloscopía estudiadas para diagnóstico de tuberculosis en el Instituto Nacional de Enfermedades Neoplásicas

\begin{tabular}{lccc}
\hline Muestra & Frecuencia & $\begin{array}{c}\text { Baciloscopía positiva } \\
\mathbf{n}(\%)\end{array}$ & $\begin{array}{c}\text { Cultivo positivo } \\
\mathbf{n}(\%)\end{array}$ \\
\hline Esputo & 77 & $10(13,0)$ & $24(31,2)$ \\
Orina & 34 & $7(20,6)$ & $6(17,6)$ \\
Heces & 11 & $2(18,2)$ & $0(0)$ \\
Lavado bronquial & 20 & $2(10,0)$ & $12(60)$ \\
Líquido pleural & 6 & $0(0)$ & $4(66,7)$ \\
Biopsia lesión sólida & 49 & $10(20,4)$ & $11(22,4)$ \\
Biopsia ganglio & 26 & $7(26,2)$ & $11(42,3)$ \\
\hline
\end{tabular}

parenquimales, linfadenopatías, efusión pleural, enfermedad miliar o atelectasia en TB primaria; y cavitación en TB postprimaria ${ }^{(3-9,19)}$. Prytz et al. describieron una serie de 91 pacientes con diagnóstico presuntivo de cáncer de pulmón que fueron sometidos a toracotomía y el hallazgo fue de TBP ${ }^{(9)}$. Pitlik et al. describen una serie de 26 casos referidos al centro de cáncer en Texas de 1973 a 1982 en los que se descartó neoplasias y se concluyó en diagnóstico de TB ${ }^{(8)}$. En nuestro estudio los hallazgos radiológicos más frecuentes fueron la presencia de nódulo pulmonar, probable infección, e imagen sugestiva de neoplasia maligna.

La localización extrapulmonar más frecuente, con excepción de la afectación pleural, en una serie de 2161 pacientes caucásicos diagnosticados con TB entre 1991 a 2008 fue la ganglionar, seguida de la urogenital y la osteoarticular, siendo el resto de localizaciones muy infrecuentes (meninges, pericardio y peritoneo) ${ }^{(17)}$. En nuestro estudio, las localizaciones extrapulmonares más frecuentes fueron la ganglionar $(21,2 \%)$, cerebral $(17,7 \%)$ y sistémica $(17,7 \%)$. La alta frecuencia de tuberculoma cerebral en nuestro estudio probablemente se debe a que estas desarrollan masas que imitan una neoplasia.

La TB ganglionar suele afectar con mayor frecuencia a niños y adultos jóvenes, y la localización más común es la cervical (63-77\%) y comúnmente es bilateral $(16,17,20)$. En nuestro estudio la mediana de edad fue 37,7 años, la localización más frecuente fue cervical y menos de $28 \%$ fueron bilaterales.

Cabe precisar que, los tuberculomas cerebrales aparecen en forma de masa sólida captadora de contraste en anillo y son comúnmente múltiples. Los lóbulos frontal y parietal son las regiones más comúnmente afectados. Tienden a confundirse con malignidad si están ausentes las características típicas como la calcificación, o cuando es de gran tamaño y causa síntomas como cefalea, vómitos, convulsiones y parálisis del nervio craneal $(16,17,20)$. Los resultados del presente estudio hallaron que la localización más frecuente fue la frontal, la mediana del diámetro de la lesión mayor fue $2,7 \mathrm{~cm}$ y se presentaron como lesiones múltiples en menos del $7 \%$.

La TB sistémica o miliar presenta un cuadro clínico muy variable, que va desde formas severas agudas que cursan con shock séptico, fallo multiorgánico y síndrome de distrés respiratorio del adulto, hasta la presentación más frecuente de evolución subaguda con síntomas insidiosos como malestar general sin deterioro del estado general $(16,17,20)$.

La TB enteroperitoneal compromete comúnmente la región ileocecal y se presenta como lesiones ulcerativas, hipertróficas y mixtas. Las imágenes muestran engrosamiento de mucosa y adenopatías mesentéricas. El compromiso peritoneal se puede observar como ascitis, masas omentales, en intestinos y en mesenterio ${ }^{(16,17,20-23)}$. Muchos casos con TB extrapulmonar tienen radiografía de tórax y baciloscopía de esputo negativos, y el problema para la obtención de muestras microbiológicas, hace que otras técnicas de imagen como TC o RM puedan ser de gran ayuda en la aproximación diagnóstica y para la obtención de muestras mediante punción ${ }^{(4,16)}$. Nosotros encontramos que siete de los casos de TB extrapulmonar tenían lesiones pulmonares y 17 baciloscopía positiva.

El $84,3 \%$ de nuestros casos presentaron un granuloma necrotizante que fue biopsiado y la mayoría se encontraron en TB ganglionar, pulmonar y sistémica. Ramírez et al. reportan que la visión microscópica de granulomas necrotizantes característicos son más frecuentes en muestras hepáticas (91-100 \%) que en medula ósea (31-82 \%) o biopsia transbronquial $(72-63 \%)^{(16)}$.

Algunos estudios describen factores de riesgo al desarrollo de TB extrapulmonar que podrían orientarnos al diagnóstico, como la edad avanzada, el sexo femenino, la raza negra y la presencia de infección por $\mathrm{VIH}{ }^{(17,20)}$. En nuestra población encontramos casi la mitad eran mujeres, un tercio tenían más de 50 años y solo dos casos fueron $\mathrm{VIH}$ positivos. 
García-Rodríguez et al. en su serie previamente comentada encuentra que la media de edad de los pacientes con TB extrapulmonar es mayor que de los pacientes con TBP (media 44,2 años vs 37,2, $\mathrm{p}<0,001$ ). Entre los pacientes que presentan TB extrapulmonar, aquellos con TB pleural (33,4 años), peritoneal $(43,7)$ o meníngea $(35,7)$ tuvieron menor edad que aquellos con afectación ganglionar $(50,6)$, osteoarticular $(58,6)$, genitourinaria $(52,1)$ y gastrointestinal $(56,1)^{(17)}$. Sin embargo, nosotros encontramos resultados opuestos, pues la TB extrapulmonar se asoció a edad menor que aquellos con TBP y aquellos con TB pleural tuvieron mayor edad que aquellos con otras localizaciones extrapulmonares en nuestro estudio. Estas diferencias se pueden deber a que nuestro criterio de selección fue imitar una neoplasia.

Finalmente, se encontró que más de la mitad de nuestros casos sugerían una neoplasia avanzada y esta interpretación puede producir la indicación de terapia solo paliativa y la no profundización en los estudios. Esta experiencia nos indica que se debe utilizar las herramientas diagnósticas de estudios de laboratorio y biopsias además del criterio clínico para llegar a un diagnóstico certero en el menor tiempo posible en los pacientes con cuadros clínicos sugestivos de neoplasia maligna.

Podemos concluir que algunos signos, síntomase imágenes asociados a TB pueden sugerir neoplasias malignas. Así, debe tenerse siempre en cuenta el diagnóstico diferencial entre cáncer y TB en zonas con alta frecuencia de estas patologías como en nuestro país.

Contribuciones de los autores: JVS, WV, LT, LC, MNB y CAC participaron en la concepción y diseño del artículo. JVS, WV y MC participaron en la recolección y obtención de resultados. JVS y CAC participaron en el análisis e interpretación de datos. JVS, CAC y VV participaron en la redacción del artículo; JVS, WV, LT, LC participaron en la revisión crítica del artículo. Todos los autores aprobaron la versión final del manuscrito.

Fuentes de financiamiento: autofinanciado.

Conflictos de interés: los autores declaran no tener conflictos de interés.

\section{REFERENCIAS BIBLIOGRÁFICAS}

1. Alarcón V, Alarcón E, Figueroa C, Mendoza-Ticona A. Tuberculosis en el Perú: Situación epidemiológica, avances y desafíos para su control. Rev Peru Med Exp Salud Publica. 2017;34(2):299-310. doi: 10.17843/rpmesp.2017.342.2384

2. Organization WH. Global tuberculosis report 2015: World Health Organization. Geneva, Switzerland: WHO; 2015.

3. Hammen I. Tuberculosis mimicking lung cancer. Respir Med Case Rep. 2015;16:45-7. doi: 10.1016/j. rmcr.2015.06.007

4. Burrill J, Williams CJ, Bain G, Conder G, Hine AL, Misra RR. Tuberculosis: a radiologic review. Radiographics. 2007;27(5):1255-73. doi: 10.1148/ rg. 275065176

5. Boyaci H, Basyigit I, Baris SA. Positron emission tomography/computed tomography in cases with tuberculosis mimicking lung cancer. Braz J Infect Dis. 2013;17(2):267-9. doi: 10.1016/j. bjid.2012.05.005

6. Shetty N, Noronha V, Joshi A, Rangarajan V, Purandare N, Mohapatra PR, et al. Diagnostic and treatment dilemma of dual pathology of lung cancer and disseminated tuberculosis. J Clin Oncol. 2014;32(6):e7-9. doi: 10.1200/JCO.2012.46.0667
7. Rolston KV, Rodriguez S, Dholakia N, Whimbey E, Raad I. Pulmonary infections mimicking cancer: a retrospective, three-year review. Support Care Cancer. 1997;5(2):90-3.

8. Pitlik SD, Fainstein V, Bodey GP. Tuberculosis mimicking cancer--a reminder. Am J Med. 1984;76(5):822-5.

9. Prytz S, Hansen J. A follow-up examination of patients with pulmonary tuberculosis resected on suspicion of tumour. Scand J Respir Dis. 1976;57(5):239-46.

10. De La Rosa GR, Jacobson KL, Rolston KV, Raad, II, Kontoyiannis DP, Safdar A. Mycobacterium tuberculosis at a comprehensive cancer centre: active disease in patients with underlying malignancy during 1990-2000. Clin Microbiol Infect. 2004;10(8):749-52.

11. Demir K, Kaymakoglu S, Besisik F, Durakoglu Z, Ozdil S, Kaplan Y, et al. Solitary pancreatic tuberculosis in immunocompetent patients mimicking pancreatic carcinoma. J Gastroenterol Hepatol. 2001;16(9):1071-4.

12. Wee A, Nilsson B, Wang TL, Yap I, Siew PY. Tuberculous pseudotumor causing biliary obstruction. Report of a case with diagnosis by fine needle aspiration biopsy and bile cytology. Acta Cytol. 1995;39(3):559-62.
13. Wee A, Nilsson B, Yap I, Chong SM. Aspiration cytology of liver abscesses. With an emphasis on diagnostic pitfalls. Acta Cytol. 1995;39(3):453-62.

14. Cakir B, Tuncbilek N, Karakas HM, Unlu E, Ozyilmaz F. Granulomatous mastitis mimicking breast carcinoma. Breast J. 2002;8(4):251-2.

15. Romand F, Gaudin JL, Bobichon R, Souquet JC. [Abdominal tuberculosis of pseudotumor aspect]. Presse medicale. 1997;26(36):1717-21.

16. Ramírez-Lapausa M, MenéndezSaldaña A, Noguerado-Asensio A. Tuberculosis extrapulmonar, una revisión. Rev Esp Sanid Penit. 2015;17(1):3-11. doi: 10.4321/S157506202015000100002

17. García-Rodríguez JF, Álvarez-Díaz $\mathrm{H}$, Lorenzo-García MV, Mariño-Callejo A, Fernández-Rial Á, Sesma-Sánchez P. Extrapulmonary tuberculosis: epidemiology and risk factors. Enferm Infecc Microbiol Clin. 2011;29(7):502-9. doi: 10.1016/j.eimc.2011.03.005

18. Von Elm E, Altman DG, Egger M, Pocock SJ, Gøtzsche PC, Vandenbroucke JP, et al. The Strengthening the Reporting of Observational Studies in Epidemiology (STROBE) Statement: guidelines for reporting observational studies. Int J Surg. 2014;12(12):14959. doi: 10.1016/j.ijsu.2014.07.013 
19. Homrich GK, Andrade CF, Marchiori RC, Lidtke GDS, Martins FP, Santos JWAD. Prevalence of benign diseases mimicking lung cancer: experience from a university hospital of Southern Brazil. Tuberc Respir Dis (Seoul). 2015;78(2):72-7. doi: 10.4046/ $\operatorname{trd}$.2015.78.2.72

20. Aisenberg GM, Jacobson K, Chemaly RF, Rolston KV, Raad, II, Safdar A. Extrapulmonary tuberculosis active infection misdiagnosed as cancer: Mycobacterium tuberculosis disease in patients at a Comprehensive Cancer Center (2001-2005). Cancer. 2005;104(12):2882-7.

21. Oge T, Ozalp SS, Yalcin OT, Kabukcuoglu S, Kebapci M, Arik D, et al. Peritoneal tuberculosis mimicking ovarian cancer. Eur J Obstet Gynecol Reprod Biol. 2012;162(1):105-8. doi: 10.1016/j.ejogrb.2012.02.010

22. Xi X, Shuang L, Dan W, Ting H, Han MY, Ying C, et al. Diagnostic dilemma of abdominopelvic tuberculosis: a series of 20 cases. J Cancer Res Clin Oncol.
2010;136(12):1839-44. doi: 10.1007/ s00432-010-0842-7

23. Ozat M, Altinkaya SO, Gungor T, Çağlar M, Zergeroglu S, Karaca M, et àl. Extraovarian conditions mimicking ovarian cancer: a single center experience of 15 years. Arch Gynecol Obstet. 2011;284(3):713-9. doi: 10.1007/ s00404-010-1705-9

Correspondencia: Carlos A. Castañeda.

Dirección: Av. Angamos Este 2520

Surquillo, Lima, Perú.

Teléfono: 992157220.

Correo electrónico:

carloscastanedaaltamirano@yahoo.com

\section{¿CÓMO ENVIAR UN ARTÍCULO A NUESTRA REVISTA?}

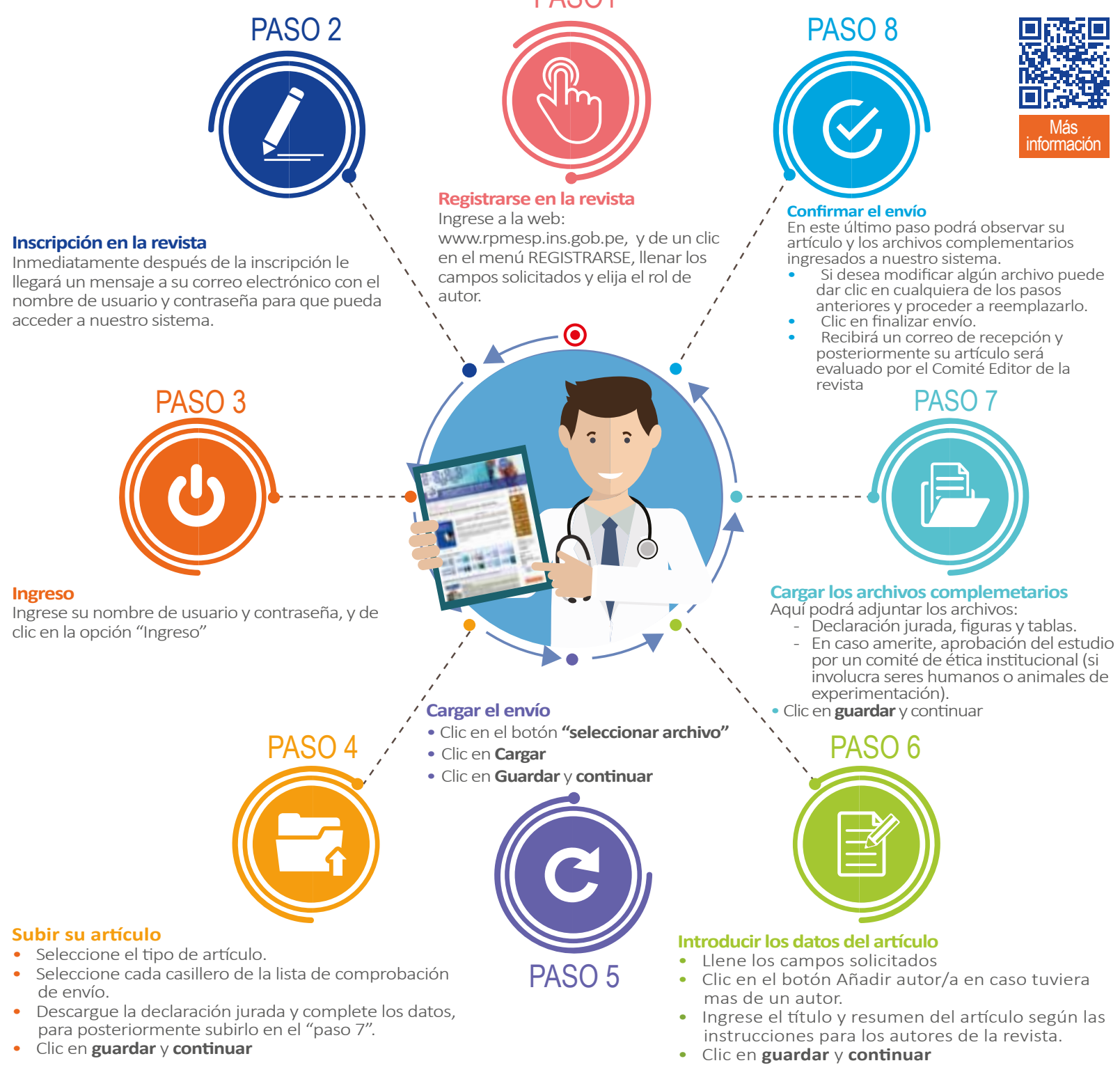

